\title{
BMJ Open Is maternity care in Scotland equitable? Results of a national maternity care
}

\section{survey}

\author{
Helen Cheyne, ${ }^{1}$ Andrew Elders, ${ }^{2}$ David Hill, ${ }^{3}$ Emma Milburn ${ }^{4}$
}

To cite: Cheyne H, Elders A, Hill D, et al. Is maternity care in Scotland equitable? Results of a national maternity care survey. BMJ Open 2019;9:e023282. doi:10.1136/ bmjopen-2018-023282

- Prepublication history and additional material for this paper are available online. To view these files, please visit the journal online (http://dx.doi. org/10.1136/bmjopen-2018023282).

Received 29 March 2018 Revised 24 September 2018 Accepted 8 October 2018
Check for updates

(C) Author(s) (or their employer(s)) 2019. Re-use permitted under CC BY-NC. No commercial re-use. See rights and permissions. Published by BMJ.

${ }^{1}$ Nursing Midwifery and Allied Health Professions Research Unit, University of Stirling, Stirling, UK

${ }^{2}$ Nursing Midwifery and Allied Health Professions Research Unit, Glasgow Caledonian University, Glasgow, UK ${ }^{3}$ Institute of Applied Health Research, University of Birmingham, Birmingham, UK ${ }^{4}$ Health and Care Analysis Division, Scottish Government, Edinburgh, UK

Correspondence to

Dr Helen Cheyne;

h.l.cheyne@stir.ac.uk

\section{ABSTRACT}

Objective High-quality maternity care is key to long-term improvements in population health. However, even within developed welfare systems, some mothers and babies experience poorer care and outcomes. This study aimed to explore whether women's experiences of maternity care in Scotland differs by their physical or sociodemographic characteristics.

Design Secondary analysis of the 2015 Scottish Maternity Care Experience Survey. The questionnaire was based on the Care Quality Commission English maternity survey.

Setting National Health Service maternity care in Scotland.

Participants The survey was distributed to 5025 women who gave birth in Scotland during February and March 2015 with 2036 respondents (41\%).

Main outcome measures The questionnaire explored aspects of care processes and interpersonal care experienced from the first antenatal contact (booking) to 6 weeks following the birth. The analysis investigated whether experiences were related to age, parity, deprivation, rurality, self-reported general health or presence of a health condition that limited daily activities. Analysis used mixed effect multilevel models incorporating logistic regression.

Results There were associations between parity, age and deprivation with gestation at booking indicating that younger women, women from more deprived areas and multiparous women booked later. Women reporting generally poorer health were more likely to describe poorer care experiences in almost every domain including continuity, pain relief in labour, communication with staff, support and advice, involvement in decision making, confidence and trust and overall rating of care.

Conclusions We found few differences in maternity care experience for women based on their physical or socioeconomic characteristics. Our findings indicate that maternity care in Scotland is generally equitable. However, the link between poorer general health after childbirth and poorer experience of maternity care is an important finding requiring further study.

\section{INTRODUCTION}

High-quality universal maternity care is recognised worldwide as essential for the safety of mothers and babies through pregnancy and birth and for long-term improvement in population health. Almost 800000

\section{Strengths and limitations of this study}

The questionnaire used has been extensively tested and used in a series of large-scale national surveys.

- A large sample based on theoretical sampling methods.

- Analysis can reveal associations but not causation.

- Differences in reported experiences could reflect variation in the quality of services received and in subjective factors such as expectations or perceptions.

- Although a large sample overall, some minority groups did not have a large enough representation to be included in the analysis.

women give birth each year in the $\mathrm{UK}^{1-3}$ making childbirth the most common reason for engagement with the National Health Service (NHS). ${ }^{4}$ For many women, pregnancy is their first adult contact with health services and, as a universal service, maternity care is often viewed as providing a unique window of opportunity to mitigate some of the factors that perpetuate health and social inequalities and to contribute to improvements in population health. This may be achieved through early identification and intervention in cases of clinical or social concerns, promotion of positive health behaviour change, provision of the information, care and support necessary for recovery from birth, and advice and support for good parenting. ${ }^{5}$

Long-standing concerns have been expressed about persistent inequalities in health, attainment and life expectancies between the most and least advantaged groups even in affluent societies. ${ }^{5-7}$ The foundations of many health and social inequalities are laid during pregnancy, childbirth and in the first days and weeks following birth. This suggests that good maternity care is particularly important for women and babies who have greater healthcare needs or increased potential for disadvantage. The findings of large-scale surveys undertaken in the UK and 
in other countries with developed welfare systems over two decades indicate that women generally report positive maternity care experiences. ${ }^{8-15}$ Yet, there is evidence that even within these healthcare systems, some groups of women consistently experience poorer quality of maternity care and outcomes. For example, secondary analyses were undertaken of the Care Quality Commission (CQC) national maternity experience surveys conducted in England in 2007 and 2010 and 2015. These analysis found that single women and women from ethnic minority groups ${ }^{16} 17$ were more likely to access services late, have poorer outcomes and report less positive care experiences, while women with self-reported disabilities experienced poorer care in relation to communication, trust, respect and support and involvement in decision making about their care. ${ }^{18}$ Similar findings have been reported in other countries. Surveys undertaken in Queensland, Australia $^{19}$ and in Sweden ${ }^{20}$ have found that younger women were less likely to report being treated with respect and kindness and being spoken to in a way that they could understand than older women ${ }^{19}$ and were less satisfied with their maternity care. ${ }^{20}$ A systematic review of population-based surveys undertaken in five countries found that immigrant women had less positive maternity care experiences than non-immigrant women. ${ }^{21}$ It appears that even in developed welfare systems, not all women's experience of care is equal.

The Institute of Medicine (IOM) quality domains of safe, effective, efficient, timely, person centred and equitable are widely accepted indicators of health service quality. ${ }^{22}$ Frequently cited in policy and care guidance, their assessment is the focus of considerable effort within the NHS (eg, refs ${ }^{23}{ }^{24}$ ). Yet, while service user's experiences of care are recognised as important, some clinicians may question their validity and relevance. ${ }^{2526}$ Historically, quality care has typically been viewed as care that is safe, effective and timely (ie, the right care in the right way at the right time) as assessed objectively, for example, through monitoring of outcomes such as adherence to evidence-based protocols, adverse incidents, referral and treatment waiting times. While these may be measured to some extent objectively, many are not routinely or reliably recorded and some, such as hospital discharge rates, are at best proxy measures for care quality.

\section{The best window on safety and quality is through the eye of the patient. ${ }^{22}$}

Across each of the IOM domains, many important elements of care quality can only be assessed by asking people about their experiences of care. This is most evident for patient centeredness with its focus on empathy, responsiveness and respect for individual need. However, being listened to and involved enough in care decisions are also important aspects of safe and effective care. Timely care is not only about procedures being undertaken as directed by guidelines, perceived accessibility of care and responsiveness of staff are equally important. The domain equitable is unique in that it cannot be assessed at the level of the individual. The IOM defines equitable as providing (across each of the other domains) care that does not vary in quality because of a person's characteristics. Therefore, equity encompasses each of the other quality domains, but it can only be assessed in relation to comparison with the experiences of others.

In this paper, we report a secondary analysis of the findings of a national survey of maternity care experience undertaken in Scotland in 2015 asking the question: is maternity care in Scotland equitable? The specific objective was to consider whether women differed in the quality of care they experienced based on age, parity, geography as defined by urban or rural dwelling, health or index of multiple deprivation.

\section{METHODS}

\section{Design}

The study involved secondary analysis of data collected in the 2015 Scottish national maternity care experience survey. The survey was undertaken as part of the Scottish Government's Care Experience survey programme. ${ }^{27}$

\section{Survey methods}

Women who gave birth in Scotland during February and March 2015 were eligible for inclusion in the survey. A stratified random sample $(60 \%)$ of these women were selected from a list of all births prepared by the NHS Central Register, using the birth registrations from the National Records of Scotland and the Community Health Index (CHI) database. The survey did not require NHS research ethics approval. Permission for access to identifiable patient data and to administer the questionnaire to mothers was granted by the Scottish Government CHI Advisory Group. Women were excluded if they were under 16 years of age or if their baby had died.

The survey was stratified by the hospital of birth (including a stratum for home births), using a disproportionate stratified sample design where some sites were oversampled to achieve the minimum number of responses required for analysis. ${ }^{28}$ The questionnaires were distributed, via an approved survey contractor, around 2 months after birth with two postal reminders. Women had the option to complete the questionnaire on paper, online or by telephone in a wide range of languages. Full details of survey methods and main results are available on the Scottish Government website. ${ }^{29}$ The survey was distributed to 5025 women with 2036 returned giving a response rate of $41 \%$. This sample size was in line with a target sample that would allow precision levels of $7 \%$ when reporting at individual hospital level. This analysis includes data from all 2036 women. The median response time was 16 weeks (range from 8 weeks to 28 weeks), with $80 \%$ of women responding between 10 weeks and 20 weeks. Respondents were more likely to be older; $10 \%$ of respondents were aged 24 years or under compared with $21 \%$ of all births in Scotland in 2015. The percentage of births that were 
first births in Scotland was 57\%, compared with 58\% in the survey. ${ }^{2}$

\section{The questionnaire}

The survey instrument (online supplementary appendix 1) was the 2013 CQC national survey of women's experiences of maternity care, ${ }^{30}$ adapted with permission, for use in Maternity Care Experience surveys in Scotland in 2013 and 2015. The questionnaire was developed by the National Perinatal Epidemiology Unit and the Picker Institute $^{31}$ and used in the English national maternity survey programme in 2007 and subsequently in 2010, 2013 and 2015. ${ }^{30}$ The questionnaire asked women to report on their experiences of maternity care at each stage of the planned maternity care journey from the first antenatal contact until 6 weeks postbirth, in relation to care process and interpersonal (relational) care. Questions addressed; access to services, appropriate care, continuity, involvement in decision making, personalised care, support and advice, communication, confidence and trust. These are enduring issues of importance to maternity service providers, policy makers and to service users $^{2324}$ and are key aspects intrinsic to each of the IOM quality domains. ${ }^{22}$ Questions on aspects of appropriate care were related to national care guidance, for example, being encouraged to move around and receiving adequate pain relief during labour. ${ }^{29}$ Changes from the CQC questionnaire, described fully in the 2013 and 2015 technical reports ${ }^{29}$ were relatively minor and reflected issues pertinent to Scottish maternity services. For example, the 2013 CQC questionnaire asked: 'If your partner or someone else close to you was involved in your care during labour and birth, were they able to be involved as much as they wanted?' For the 2015 Scottish survey, this question was extended to include: 'were they able to stay with you as much as you wanted?' as many maternity units were starting to offer opportunities for women's partners to stay in the maternity hospital overnight following the birth. A new question was added about contacts with the health visitor (public health nurse) involvement reflecting the newly introduced health visiting pathway for Scotland. The questions on personal and demographic characteristics and general health were also specific to the Scottish questionnaire. These questions were standardised across all of the Scottish Government patient experience surveys. This section asked about health: 'How would you rate your health in general?' and 'Do you have a physical or mental health condition or illness lasting or expected to last 12 months or more?' with a follow-up question 'Does your condition or illness reduce your ability to carry-out day-to-day activities?' and questions about religion, sexuality and ethnicity. A significant change to the CQC 2013 questionnaire was the inclusion of a rating of care question. While the focus of the questionnaire was on experience, rather than satisfaction with care, women were asked to rate their care overall for hospital care (antenatal, intrapartum and postnatal) and community-based postnatal care. The distinction between these concepts is that rating seeks a judgement about care quality, that is, my care was good or not good, based on both experience and expectation. Questions about experience ask for a description of specific aspects of care received, leaving the judgement about what constitutes 'good care' to those who design and analyse the questionnaire. For example, the question 'were you given enough time to ask questions or discuss your pregnancy?' assumes that being given enough time is an important element of good care.

We grouped survey questions into outcome subsets for each dimension of care quality described above, under the overall categories of care process and interpersonal care, along with general rating of care. Table 1 indicates how the groupings broadly correspond to almost all of the IOM domains of healthcare quality. ${ }^{22}$ Each subset included a suite of questions most relevant to that subset, although as with the quality domains, some questions could have applied to more than one subset. General rating of care differs as described above, in that it brings together experience and expectation and does not correspond specifically with the IOM quality domains.

\section{Patient involvement}

Service users were not directly involved in this study. However, maternity service users contributed to the

Table 1 Classification of questionnaire items and their relationships with Institute of Medicine (IOM) domains

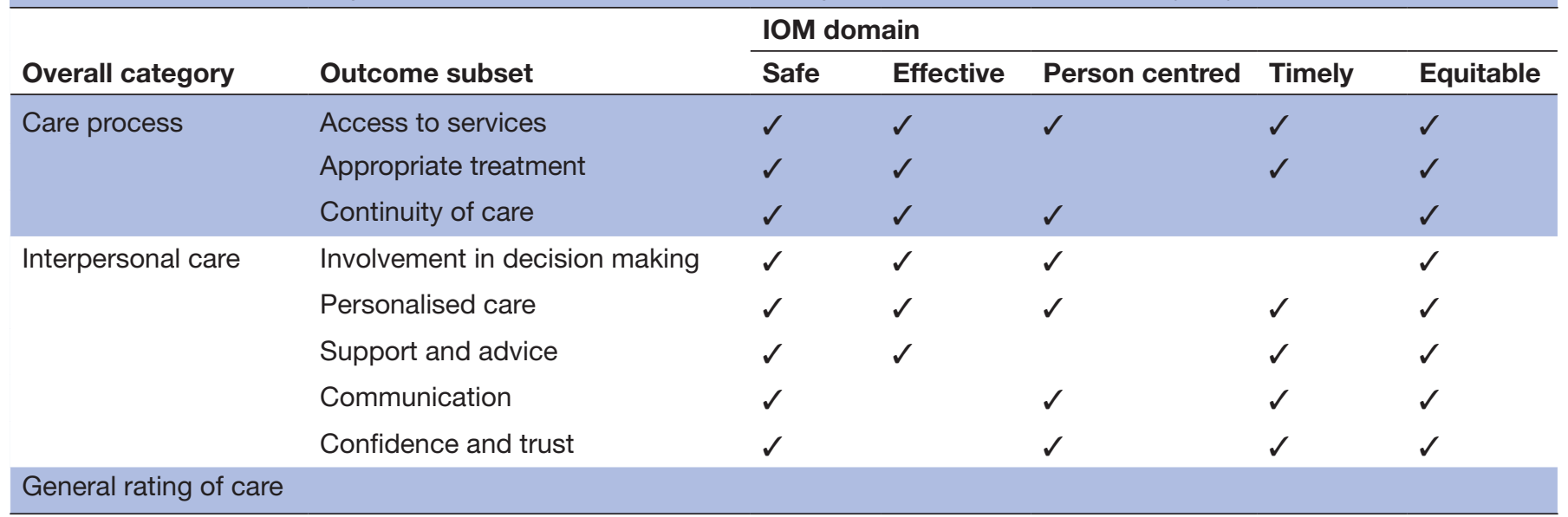


adaptation of the national maternity survey questionnaire through a survey advisory group that included an NHS Board Maternity Service Liaison Committee.

\section{Statistical analysis}

The purpose of the analysis was to investigate whether maternity care outcomes are dependent on demographic features (independent variables), specifically age in years, parity (primiparous or multiparous), deprivation, rurality, general health and the presence of health conditions or illnesses perceived to limit the ability to carry out day-to-day activities (limiting condition). Deprivation percentiles from the Scottish Index of Multiple Deprivation $2014^{32}$ were used as a proxy measure for socioeconomic status based on the postcode of the participant, where 1 is the most deprived and 100 is the least deprived. Rurality was based on the Scottish Government Urban/ Rural Classification, ${ }^{33}$ which measures the size of population and remoteness of the settlement of residence on an ordinal scale of 1 for most urban to 6 for most rural. Self-reported general health was categorised on an ordinal scale as (1) 'very good', (2) 'good' or (3) 'fair, bad or very bad', and self-reported presence of limiting conditions or illnesses was categorised on an ordinal scale as (1) no limiting condition, or (2) a limiting condition resulting in reduced activity either 'never', (3) 'a little' or (4) 'a lot'. Age and deprivation were treated as continuous variables. Demographic variables with nominal categories (sexual orientation and ethnicity) were not included in the analysis because of very small numbers in all categories apart from heterosexual $(96.8 \%)$ and white $(92.3 \%)$.

In order to account for potential bias resulting from the stratified design of the survey, ${ }^{28}$ weights were applied to the survey responses, defined as the ratio of expected to observed numbers of responses. For this purpose, each outcome was categorised as either a hospital or community outcome. Observations for hospital outcomes from women attending under-represented hospitals were assigned a higher weight and, similarly, observations from over-represented hospitals were assigned a lower weight. For the weighting of community outcomes, an additional level was incorporated to allow for health board of residence. All analyses were adjusted for survey weights.

The outcome responses, independent variables and weights were analysed from a single cross-sectional dataset. The format of the outcome variables were variously binary (yes/no), nominal or ordinal. Where the survey had ordinal categorisations such as 'Yes, always', 'Yes, nearly always', 'No, almost never' and 'No, never', these questions were dichotomised to yes/no binary outcomes by making the first two in this example to simply 'Yes' and the latter two to 'No'. For nominal outcomes, responses were converted into separate binary (yes/no) outcomes. Responses recorded as 'don't know' were treated as missing and excluded from the analyses.

We carried out a prespecified plan to use linear mixed effect multilevel models with demographic covariates fitted as fixed effects and hospital (for hospital outcomes) or health board level (for community outcomes) fitted as random effects. In this way, variability at the hospital or health board level was taken into account when estimating the effect of independent variables. Analyses were carried out to examine the sensitivity of fitting geographic variables as either fixed or random effects. Dichotomous responses were analysed using binary logistic regression and ordinal outcomes with ordered logistic regression, with ORs estimated with $95 \%$ CIs for all outcomes. Penalised likelihood logistic regression was used if there was perfection prediction. ${ }^{34}$ Significance was determined to be at the 0.05 level, with no adjustment for multiple testing being considered necessary given the multilevel models used. ${ }^{35}$ All analyses were conducted using Stata (StataCorp 2015. Stata Statistical Software: Release 14. College Station, TX: StataCorp LP).

\section{RESULTS}

For each outcome subset, we report here any statistically significant results relating to the independent variable with the largest number of significant associations. Although we highlight these findings in this section, we emphasise however that the majority of the effect estimates are not statistically significant indicating that the majority of maternity care outcomes explored were not dependent on demographic features.

\section{Care process}

For access to services (table 2), parity had the highest number of statistically significant associations. Evidence indicated that women who were having a baby for the first time (primiparous) had better access. Primiparous women saw their first health professional earlier than multiparous women (OR 1.65, 95\% CI 1.23 to 2.21) and had their first antenatal care assessment (booking) earlier (OR 1.19, 95\% CI 1.01 to 1.40). Other demographic factors, in addition to parity, were associated with time of booking, with older women booking earlier (OR 0.98, $95 \%$ CI 0.96 to 0.98 , including adjustment for parity) and women in least deprived areas also booking earlier (OR $1.002,95 \%$ CI 1.000 to 1.004$)$. There was no association with either general health or having a limiting condition or illness.

General health was the main factor influencing appropriateness of treatment received (table 2) indicating that women in better health described better experiences of quality of care. During their labour, compared with women in good health, women who reported fair, bad or very bad health felt that they had been less able to choose the most comfortable position for themselves (OR $0.37,95 \%$ CI 0.24 to 0.58 ) and that they experienced less support with pain management (OR $0.48,95 \%$ CI 0.26 to $0.90)$. Immediately after giving birth, these women were less likely to have skin-to-skin contact (OR $0.35,95 \%$ CI 0.20 to 0.64 ) with their baby. During postnatal care fewer women reporting fair, bad or very bad general health felt informed about the need for a maternal postnatal 


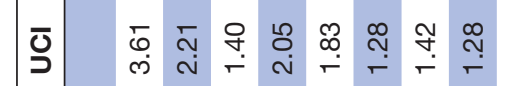

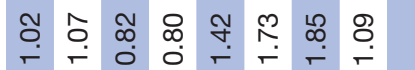

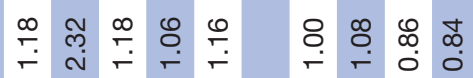

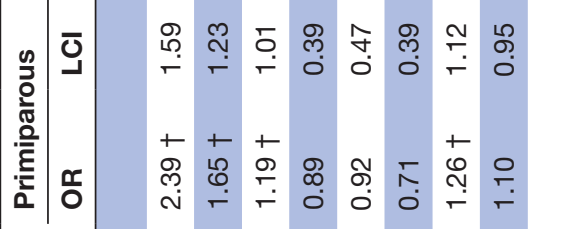

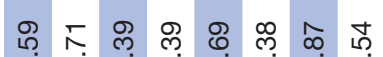

० 0 Oे

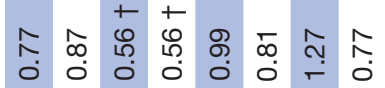

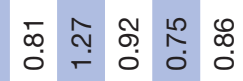

$5 \sqrt{5} \frac{0}{2}$

$\begin{array}{llll}1 & 0 & 0 & 0 \\ 0 & 0 & 0\end{array}$

ర

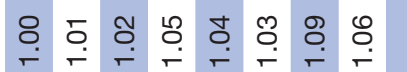

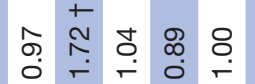

$\begin{array}{llll}\infty & \infty & 1 & \hbar \\ \infty & \infty & 0 & 0 \\ 0 & 0 & 0 & 0\end{array}$

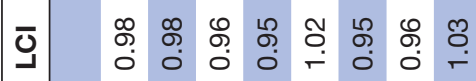

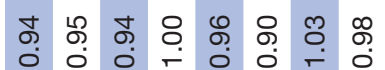

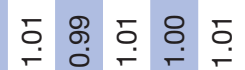

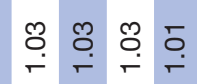

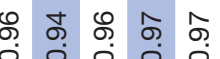

৪

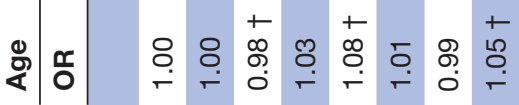

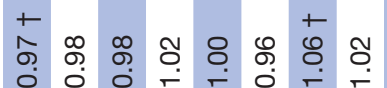

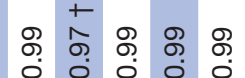

ธ두 웅 मुڤ

$\overline{\mathrm{O}}$

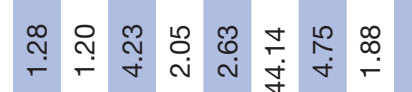

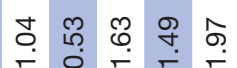

ㄷํำ 욷

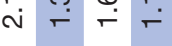

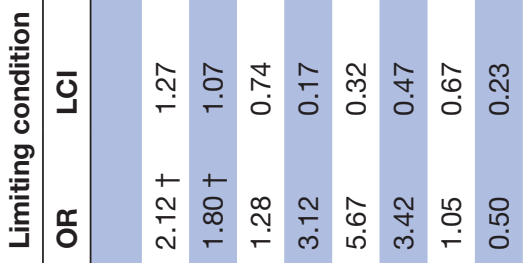

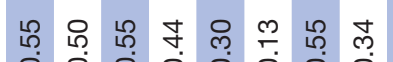

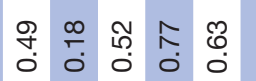

กิน

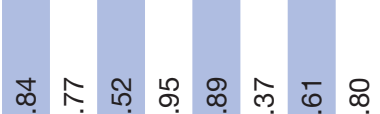

กั่

$\begin{array}{llll}\hat{0} & 0 & \infty & 0 \\ \circ & 0 & 0 \\ 0 & 0 & 0\end{array}$

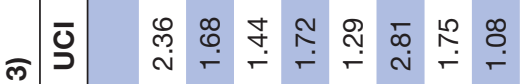

$\stackrel{\infty}{\infty}$

菅

œ

원 告

苞

吾

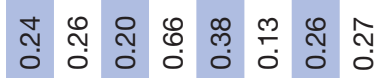

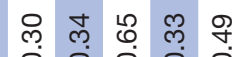

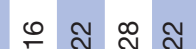

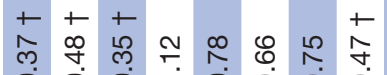

ז.

ț

离

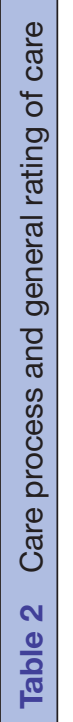

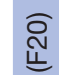

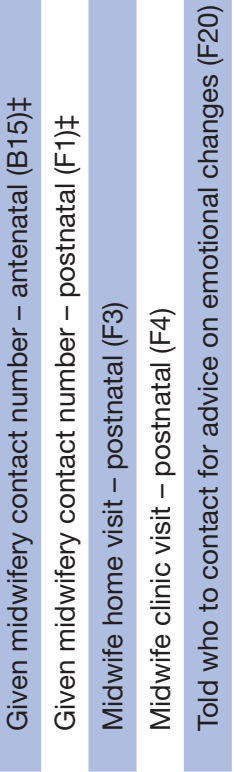

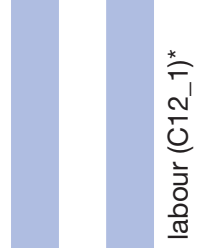

तु

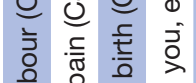

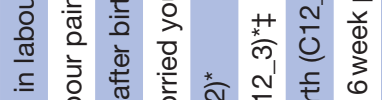

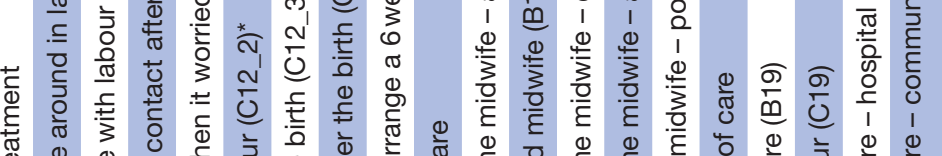

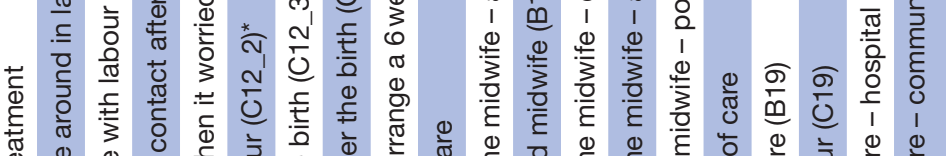

离

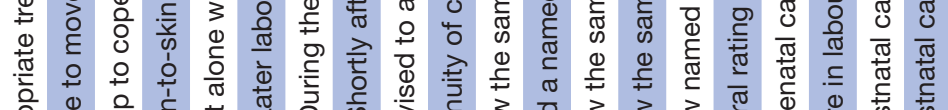

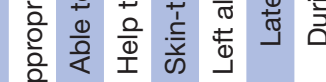

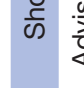
垔 脑

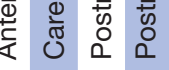

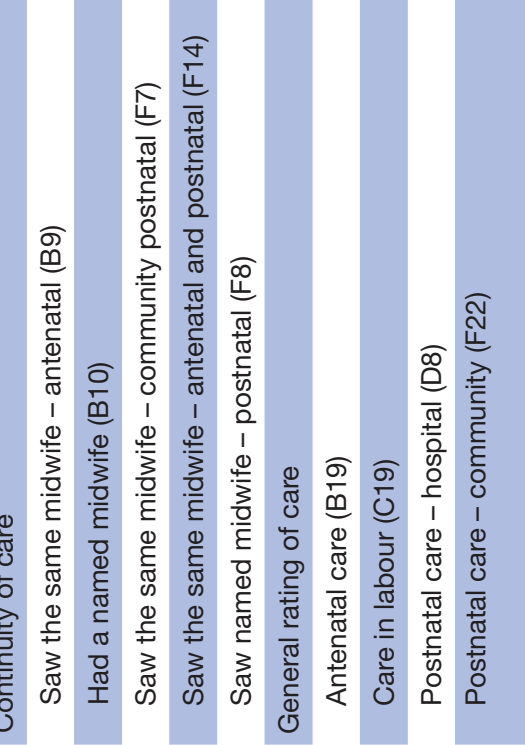

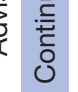




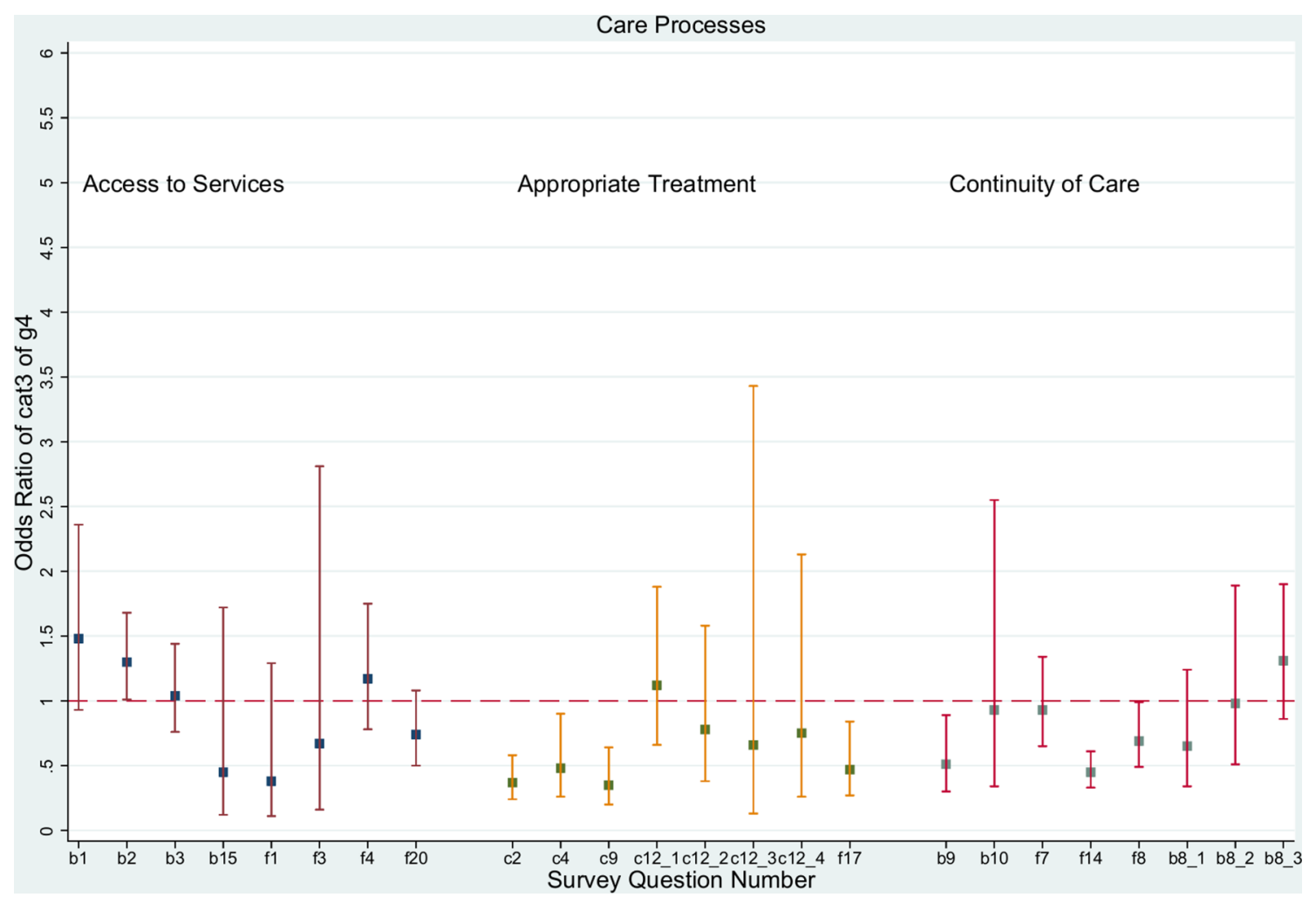

Figure 1 This shows $\mathrm{OR}$ and $\mathrm{Cl}$ for a given question in each of the three care process groups of access to services, appropriate treatment and continuity of care. Where the $\mathrm{Cl}$ lies entirely above the $\mathrm{y}=1$ line, experience is rated as positive (good care) for that question or negative (poor care) if it lies entirely below that line.

check-up (OR $0.47,95 \%$ CI 0.27 to 0.84 ). There were no associations with either limiting illness or deprivation.

Although all factors had a link with at least one continuity outcome (table 2), general health had the highest number of significant associations. Fewer women in poorer general health saw the same midwife at each antenatal appointment (OR $0.51,95 \%$ CI 0.30 to 0.89 ), fewer had the same midwife for both antenatal and postnatal care (OR $0.45,95 \%$ CI 0.33 to 0.61 ) and fewer saw their named midwife postnatally (OR $0.69,95 \%$ CI 0.49 to 0.99) (figure 1).

\section{Interpersonal care}

There were many communication outcomes (table 3) for which a strong association was observed with general health, indicating that less healthy women experienced worse communication from maternity service providers (figure 2). Only general health and age showed any associations with confidence and trust (table 3 and figure 2). Women with poorer health reported that they had experienced less respect and dignity during labour (OR 0.44 , $95 \%$ CI 0.24 to 0.83 ) and had less confidence and trust in the midwifery team after discharge (OR $0.29,95 \%$ CI 0.18 to 0.70 ). Conversely, older mothers had more confidence and trust both in the staff providing care during labour (OR 1.06, 95\% CI 1.01 to 1.11 ) and the midwifery team after discharge (OR 1.10, 95\% CI 1.05 to 1.15) (table 3).

Parity had the greatest amount of association with involvement in decision making (table 3). Primiparous women were more likely to report that they were offered a choice of hospital (OR 1.34, 95\% CI 1.11 to 1.61 ), a choice of giving birth in midwife led unit or birth centre (OR 1.84, 95\% CI 1.49 to 2.26) and the choice of having a home birth (OR 1.71, 95\% CI 1.31 to 2.24). Mothers who had not previously had a baby also felt more sufficiently informed in order to make a decision about where to give birth (OR 1.35, 95\% CI 1.08 to 1.67). However, first-time mothers reported that they were less able to involve a birth partner as much as they wanted (OR 0.52, 95\% CI 0.35 to 0.77 ). General health and deprivation also had some associations with decision-making outcomes but not limiting illness or age.

For personalised care outcomes (table 3), there was a small amount evidence of dependency on all factors (except limiting illness), with general health (figure 3) and parity having the most number of significant associations. Women in poorer health were less likely to have had their personal circumstances taken into account at antenatal consultations $(0.22,95 \%$ CI 0.08 to $0.58)$ and, when they were in hospital, they reported that their partners were more restricted to visiting hours (OR $1.54,95 \%$ CI 1.08 to 2.17 ). The partners of primiparous women were also more restricted to visiting hours (OR $1.72,95 \%$ CI 1.23 to 2.38 ), and these women felt that their decisions about feeding were respected less (OR 0.6, $95 \%$ CI 0.41 to 0.87 ). 


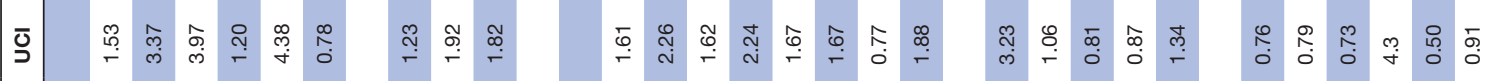

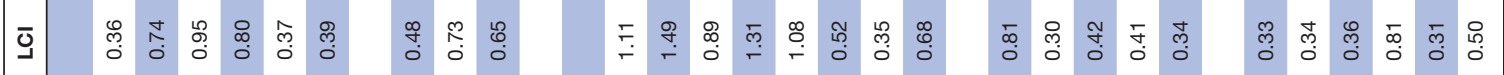

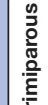

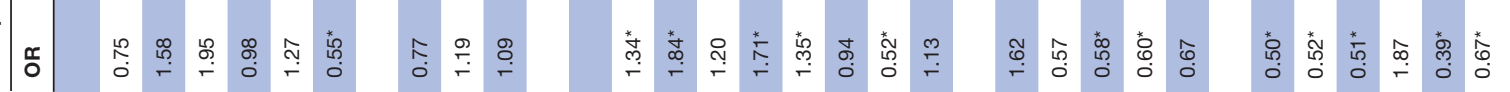

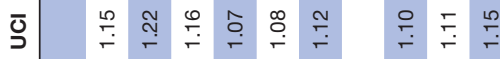

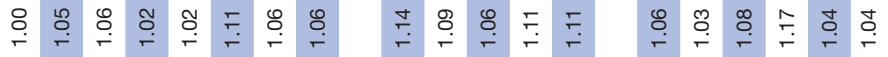

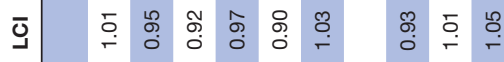

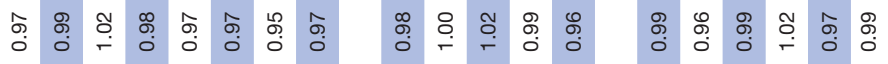

d

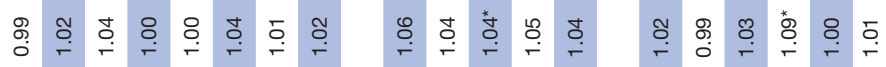

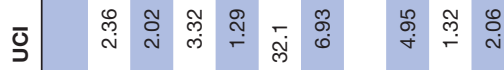

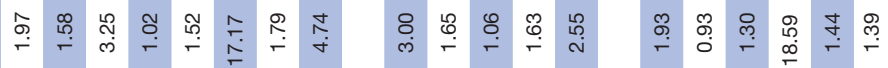

产

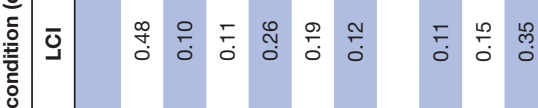

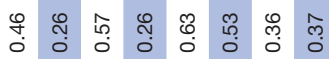

ז.

㑊

䜿

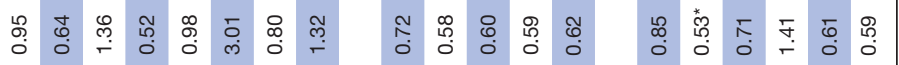

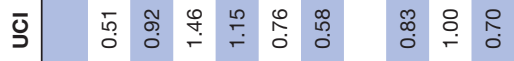

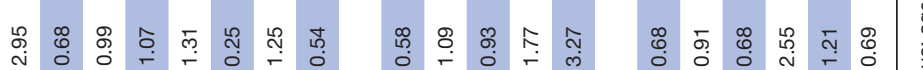

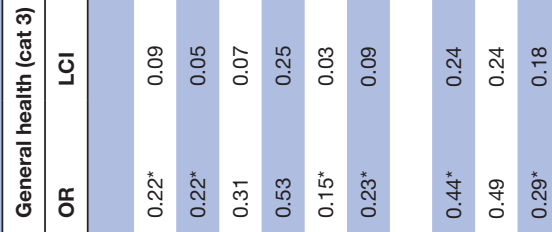

雚

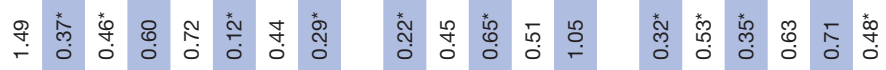

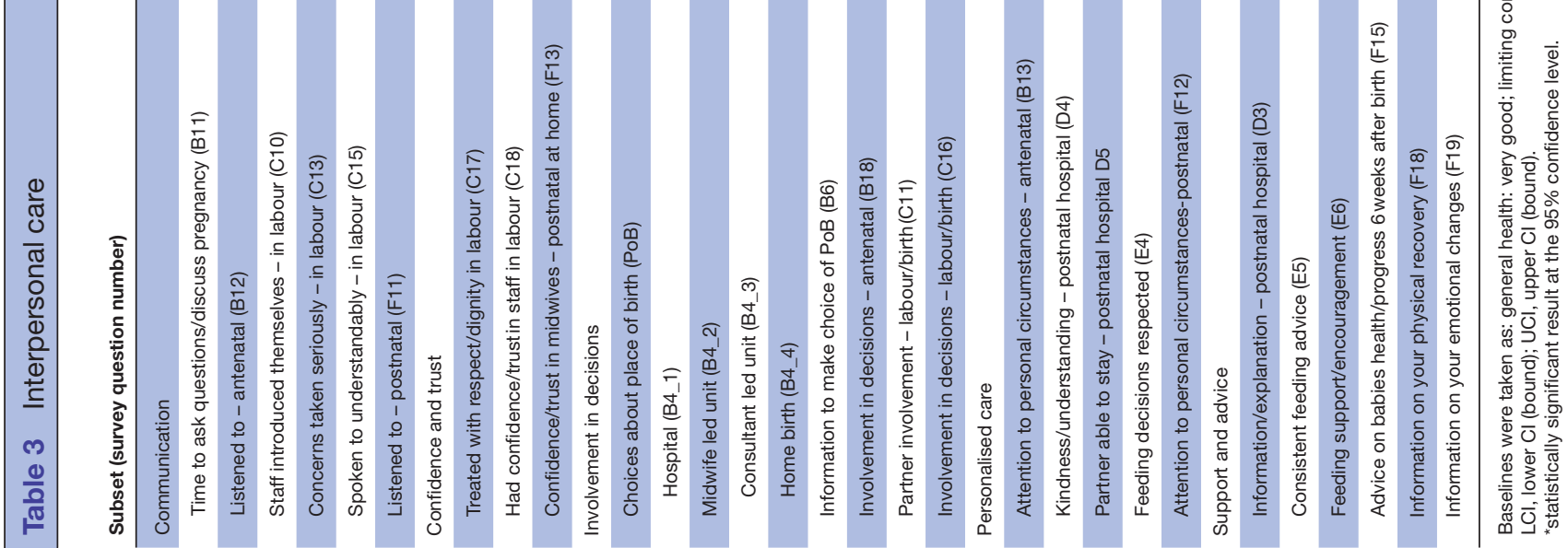




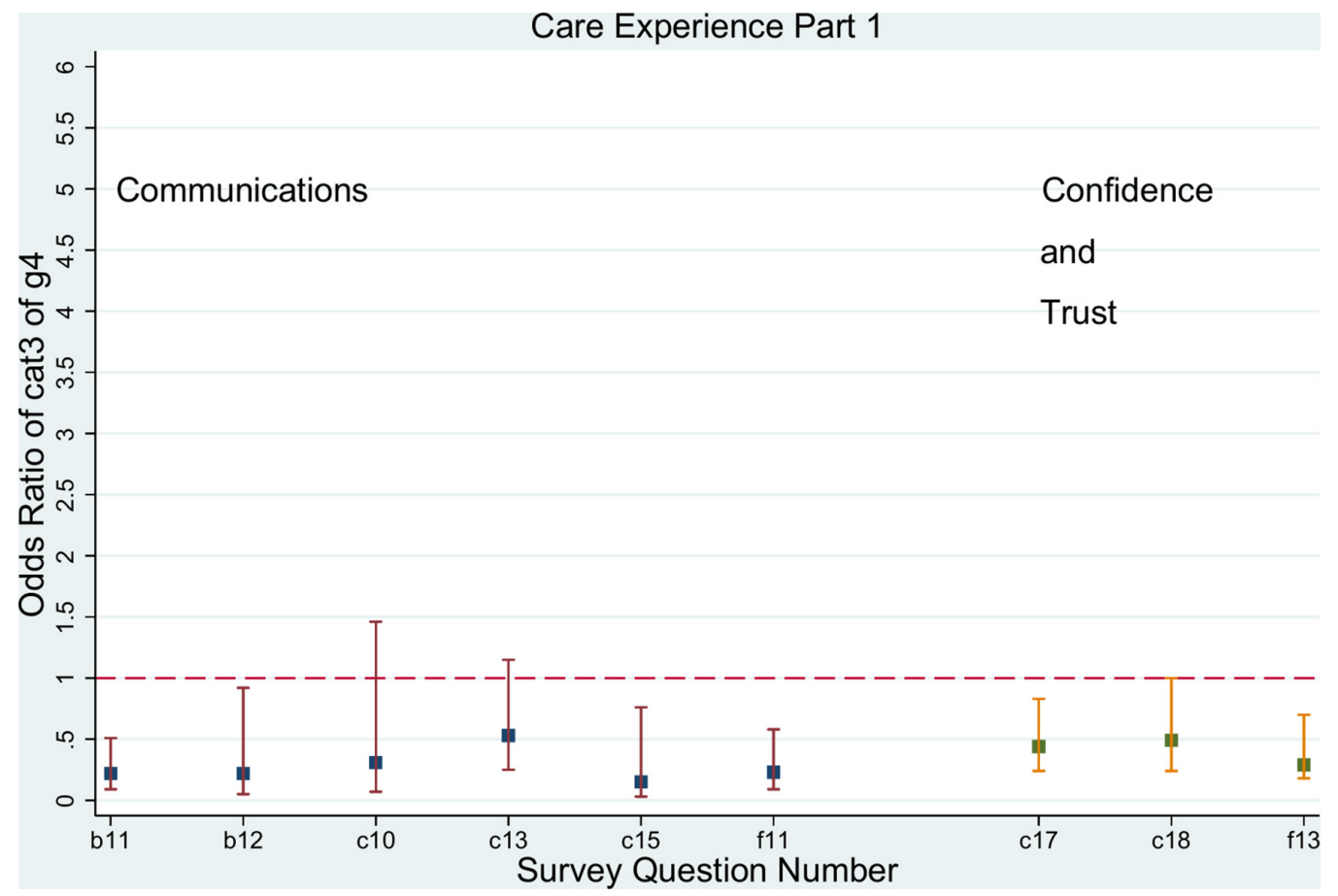

Figure 2 This shows ORs and Cls for a given question in each of the first part of the care experience groups, namely communications and confidence and trust. Where the $\mathrm{Cl}$ lies entirely above the $\mathrm{y}=1$ line, experience is rated as positive (good care) for that question or negative (poor care) if it lies entirely below that line.

All factors had some association with support and advice outcomes (table 3), with parity having the most number of associations. Women with worse health were less likely to receive sufficient information following birth (OR $0.32,95 \%$ CI 0.16 to 0.68 ), received less consistent feeding advice (OR $0.53,95 \%$ CI 0.31 to 0.91 ) and less support and encouragement about feeding (OR 0.35, $95 \%$ CI 0.18 to 0.68 ) (figure 3 ). Mothers in poorer general health also reported receiving less sufficient information postnatally regarding both their physical recovery (OR $0.71,95 \%$ CI 0.42 to 1.21$)$ and their emotional recovery (OR $0.48,95 \%$ CI 0.34 to 0.69 ) (table 3 ).

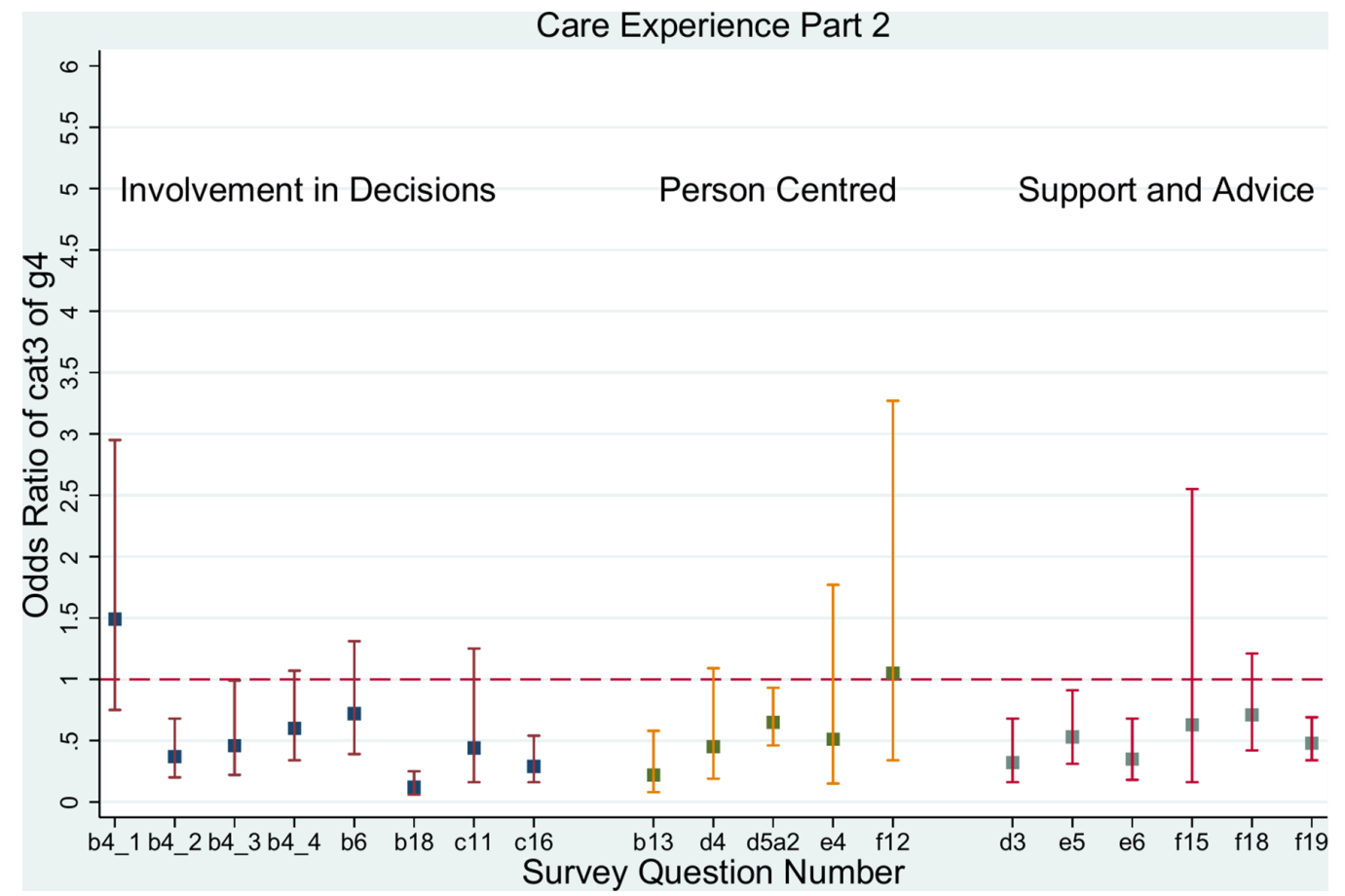

Figure 3 This shows $\mathrm{OR}$ and $\mathrm{Cl}$ for a given question in each of the second part of the care experience groups, namely involvement in decisions, person centred and support and advice. Where the $\mathrm{Cl}$ lies entirely above the $\mathrm{y}=1$ line, experience is rated as positive (good care) for that question or negative (poor care) if it lies entirely below that line. 


\section{General rating}

Only general health and parity showed any associations with general ratings of care (table 2), with health status having more significant associations. Women with poorer health provided worse ratings for each stage of their maternity care journey: antenatal care (OR 0.26, 95\% CI 0.16 to 0.40 ), care during labour (OR $0.32,95 \%$ CI 0.22 to 0.47 ), postnatal hospital care (OR $0.43,95 \%$ CI 0.28 to 0.67 ) and postnatal care at home (OR $0.36,95 \%$ CI 0.22 to 0.59 ).

The sensitivity analyses to examine geographic variables either as fixed or random effects produced very similar results, with only minor differences relating to rurality (there were slightly fewer significant associations between rurality and outcomes when geographic variables were fitted as random effects).

\section{DISCUSSION}

This paper reports on the results of a reanalysis of data from a national survey of women's maternity care experiences that aimed to examine associations between women's demographic characteristics and their experiences of maternity care. The characteristics included were: age, parity (first or subsequent birth), socioeconomic deprivation, urban or rural dwelling, general health and presence of a health condition that limits daily activities.

The survey included questions about women's experiences of key aspects of the process of care they received including early access to services, knowing who to contact in case of concerns, being given adequate pain relief during labour and receiving continuity of care. Relational aspects of care experience included important questions about communication between women and their care providers, receiving the advice and support that they needed and being involved in decisions about their care. These are all important indicators of care that is safe, effective, person centred and timely, and all are best assessed through directly asking the recipients of maternity care. Across most of the outcomes and demographic characteristics examined, there were few consistent associations identified. This means that there was no evidence that women's experience of maternity care in Scotland varied systematically by age, parity, socioeconomic status, geography as defined by urban or rural dwelling or having a health condition that limited daily activities. This suggests that, in general, maternity care in Scotland is equitable.

However, there were some important exceptions. Women living in the most deprived areas booked for their antenatal care significantly later than women living in the least deprived areas. These women were also less involved in aspects of decision making about their care. In contrast, first time mothers tended to access care earlier and feel more involved in choices and care decisions; however, these women had poorer experiences in some aspects of person-centred care in particular in relation to involvement of their partner during labour and postnatal care in hospital. There were some associations across all aspects of personalised care and support and advice indicating that some groups of women experienced poorer care for at least some aspects of these domains. However, there was no evidence of consistent or systematic detriment experienced by any particular group.

The clear exception and most striking finding was that women who reported having poorer general health also reported consistently poorer experiences of care. This was apparent across all outcome areas and across the majority of questions within each of the themes with the exception of access to services. Women who had poorer general health also experienced less continuity of care, had less positive care experiences during labour and birth, including pain management, and being treated with respect and dignity. This group of women had less confidence and trust in staff, felt less involved in decisions about their care and experienced poorer communications with staff. They also felt that their infant feeding decisions were not respected, that they received less support and advice in the postnatal period and they were more likely than women reporting good health to report receiving enough information about their own physical or emotional health in the 6 weeks after giving birth. It is interesting that these associations were consistent and evident across all of the domains explored, while there were no similar findings for women who reported having a condition or illness that limited their daily activities. Caution, however, should be exercised when interpreting these results, given the multiplicity of analyses and potential for some chance findings.

The maternity survey national report found that women were generally highly satisfied with their care, and this reanalysis has found that this did not vary across the demographic or health dimensions examined with the exception of women who reported poorer general health. This fits with the findings of other studies of maternity care satisfaction that have indicated that women are generally satisfied with the care they have received and suggests that experience broadly matches expectations. ${ }^{36} 37$

The findings of this study do not fully reflect the findings of other similar studies. Several studies have found that women from ethnic minority groups or immigrant women had poor experiences of care, ${ }^{16} 1721$ for example, that they accessed services later, experienced less effective pain relief during labour and had poorer experiences of interpersonal care. The current study was not able to include ethnicity in the analysis due to smaller overall sample size and the very low numbers of women from minority groups completing the survey. This partly reflects that Scotland is not so ethnically diverse as other countries in the UK and Europe; however, the maternity survey also received proportionately fewer responses from women in minority groups. More focused research is needed to understand the maternity care experiences of women from ethnic minority groups in Scotland.

This study differed from the findings of several studies reporting inequalities in care experienced 
by younger women and women from lower income groups. ${ }^{16} 1920$ This study found no consistently negative associations with age or socioeconomic status. There has been considerable effort through Scotland's health and social care policies over the last decade $^{38}$ to reduce the impact of inequality. Furthermore, improving early access to maternity care for women across different levels of deprivation was an NHS Scotland national performance target up until 2015 when evidence indicated that the target had been achieved ${ }^{39}$ The findings of this study suggest that to some extent maternity services have been successful in providing equitable care to the demographic groups that were traditionally considered less likely to engage with maternity services: younger women and women from poorer areas. While this study found that women from lower socioeconomic groups continue to access services significantly later than women from higher socioeconomic groups, this was still within the national target of antenatal booking by 12 weeks of pregnancy. There was no evidence of poorer care experience for these women across the other dimensions studied.

The clear finding that women who had poorer general health tended to report poorer care experiences is consistent both with other analysis of the Scottish Care Experience surveys ${ }^{4041}$ and wider studies that have explored the influence of health status. ${ }^{42}$ Although this finding has not been reported in other similar studies of maternity care experience, this may in part be due to differences in health characteristics included the survey instruments. For example, although Scottish survey is based on the CQC questionnaire, the demographic and health questions differ. While the CQC questionnaire contains a range of options relating to long-standing health conditions and disability, ${ }^{18}$ the Scottish survey included only three questions relating to general health and longterm physical or mental illness. These differences may limit comparability of findings between studies.

\section{Strengths and limitations}

A strength of the study is that the questionnaire used was based on the English National Survey questionnaire. This was subject to rigorous cognitive and field testing and successfully used in a series of large-scale English national surveys prior to its use in the Scottish national survey. ${ }^{31} 32$ A rigorous stratified random sampling strategy was used, and this included all maternity units and all NHS Boards in Scotland resulting in a sample likely to be to largely representative of the maternity population in Scotland. However, large-scale surveys have some limitations related to design and methods. The response rate to the survey was low at $41 \%$, and younger women were under-represented among respondents potentially limiting the generalisability of the findings. However, weighting and the rigorous analytical techniques employed accounts for these issues to some extent. The questionnaire contains a mixture of closed, structured questions and four open questions where women were able to provide more context to their responses. These open comments were analysed and presented alongside the quantitative data in the national report for the survey. ${ }^{29}$ However, the structured wording does limit our understanding of findings. In particular, wording of the general health question means that it is not possible to say whether women experienced poorer general health as a result of experiencing poorer maternity care, or whether women who had poorer general health then experienced poorer maternity care. While there is a clear association between poorer general health and poorer care experience, cause and effect cannot be inferred. This will be addressed in future iterations of the Scottish national maternity survey. Small numbers of women in some demographic groups, in particular, for ethnic groups, meant that it was not possible to include this factor in the analysis. This may mean that that some important areas of inequity have not been uncovered. In common with most large -scale maternity experience surveys, the Scottish maternity experience survey was targeted specifically at women who had recently used maternity care. While a small number of questions addressed the involvement of partners, these do not address the important issue of partner, or wider family experience of involvement in maternity care or care needs in the early weeks and months following birth. This is an issue that should be addressed in future research.

The findings of this study indicate that women's experience of maternity care matters. There is a clear association between poorer general health at 2 months postbirth and poorer experience of maternity care. This association was found across almost all dimensions of care process and interpersonal care examined indicating that women who report poorer general health also are more likely to experience poorer quality of maternity care across all of the IOM quality dimensions. Further research is required to explain this finding. Data linkage and longer term follow-up studies that link women's experiences of maternity care with subsequent health and well-being outcomes is also required to more fully explore the links between maternity care experience and longer term health and well-being.

Maternity care clinicians need to be aware of the potential long-term impacts of poor care experiences on women's general health and to understand that women's experience of care provides important indicators of care that is safe, effective and equitable as well as person centred and should seek to adopt models of care that have been demonstrated to be effective in improving women's experiences across these domains such as continuity of care. ${ }^{43}$

Well-conducted surveys of the healthcare experience should be considered central to patient safety programmes and not considered to be merely "nice to have' but secondary to measures traditionally considered to be more objective. The findings of this study 
suggest that there are previously unrecognised areas of concern relation to women who report poorer general health following childbirth. Although this analysis could not establish whether these women experience poorer health as a result of experiencing poorer quality maternity care or if their poorer general health resulted in them experiencing poorer care, either option is concerning, and the needs of these women require further research.

Acknowledgements Thanks are due to the women who completed the Scottish National Maternity Experience Questionnaire.

Contributors $\mathrm{HC}$ initiated the study, contributed to the analysis plan and drafted the paper. AE led the development of the analysis plan and contributed to the analysis and drafted the results section of the paper; DH contributed to the analysis plan, undertook the analysis, produced the tables and figures and commented on the paper; and EM led the national survey planning and fieldwork, contributed to the analysis plan and commented on drafts of the paper. All authors contributed to revisions of the paper following peer review.

Funding This work was supported by funding from the Scottish Government Health and Social Care Analysis Division.

Competing interests None declared.

Patient consent for publication Not required.

Ethics approval Scottish Government CHI Advisory Group.

Provenance and peer review Not commissioned; externally peer reviewed.

Data sharing statement The data for the study are from the 2015 Scottish Maternity Care Survey and were shared by the Scottish Government Health and Social Care Analysis Division with the Nursing, Midwifery and Allied Health Professions Research Unit for the purposes of producing this analysis. More information about the survey can be found here: www.gov.scot/maternitysurvey.

Open access This is an open access article distributed in accordance with the Creative Commons Attribution Non Commercial (CC BY-NC 4.0) license, which permits others to distribute, remix, adapt, build upon this work non-commercially, and license their derivative works on different terms, provided the original work is properly cited, appropriate credit is given, any changes made indicated, and the use is non-commercial. See: http://creativecommons.org/licenses/by-nc/4.0/.

\section{REFERENCES}

1. Office for National Statistics (ONS). Statistical Bulletin: Births in England and Wales. ONS Website. 2015 https://www.ons.gov. uk/peoplepopulationandcommunity/birthsdeathsandmarriages/ livebirths/bulletins/birthsummarytablesenglandandwales/2015 (Accessed 24 Sep 2017).

2. Information Services Division (ISD). Scotland. Births in Scottish Hospitals. ISD Scotland website. http://www.isdscotland.org/HealthTopics/Maternity-and-Births/Births/ (Accessed Sep 2017).

3. Northern Ireland Statistics and Research Agency. Registrar General Annual Report. Northern Ireland Statistics and Research Agency website. https://www.nisra.gov.uk/statistics/births-deaths-andmarriages/registrar-general-annual-report (Accessed 24 Sep 2017).

4. National Audit Office (NAO). Maternity services in England. NAO website. http://www.nao.org.uk/2013-maternityservices (Accessed 24 Sep 2017).

5. Department for Health and Social Care. Independent report. Chief Medical Officer's annual report 2012: Our Children Deserve Better: Prevention Pays. GOV.UK (UK Government) website. https://www. gov.uk/government/publications/chief-medical-officers-annualreport-2012-our-children-deserve-better-prevention-pays (Accessed 24 Sep 2017).

6. Royal College of Paediatrics and Child Health $(\mathrm{RCPCH})$. State of Child Health Report 2017. Child health in jeopardy due to an alarming gap between rich and poor. RCPCH website. http://www. rcpch.ac.uk/state-of-child-health (Accessed 24 Sep 2017).

7. Save the Children. State of the World's Mothers 2015. The Urban Disadvantage. 2015 www.savethechildren.org/content/dam/usa/ reports/advocacy/sowm/sowm-2015.pdf (Accessed July 10, 2018).

8. Redshaw M, Rowe R, Hockley C, et al. Recorded delivery: a national survey of women's experience of maternity care. Oxford: National Perinatal Epidemiology Unit, 2006.
9. Redshaw M, Heikkilä K. Delivered With Care. A National Survey of Women's Experience of Maternity Care. Oxford: National Perinatal Epidemiology Unit, 2010.

10. Redshaw M, Henderson J. Safely delivered: a national survey of women's experience of maternity care 2014. Oxford: National Perinatal Epidemiology Unit, 2015.

11. Henderson J, Redshaw M. Change over time in women's views and experiences of maternity care in England, 1995-2014: A comparison using survey data. Midwifery 2017;44:35-40.

12. Brown SJ, Davey MA, Bruinsma FJ. Women's views and experiences of postnatal hospital care in the Victorian Survey of Recent Mothers 2000. Midwifery 2005;21:109-26.

13. Hildingsson I, Rådestad I. Swedish women's satisfaction with medical and emotional aspects of antenatal care. $J$ Adv Nurs 2005;52:239-49.

14. Wiegers TA. The quality of maternity care services as experienced by women in the Netherlands. BMC Pregnancy Childbirth 2009;9:18.

15. Public Health Agency of Canada. What mothers say: the Canadian maternity experiences survey. 2009. Ottawa http://www.publichealth. gc.ca/mes (Accessed $10 \mathrm{Jul}, 2018$ ).

16. Raleigh VS, Hussey D, Seccombe I, et al. Ethnic and social inequalities in women's experience of maternity care in England: results of a national survey. J R Soc Med 2010;103:188-98.

17. Henderson J, Gao H, Redshaw M. Experiencing maternity care: the care received and perceptions of women from different ethnic groups. BMC Pregnancy Childbirth 2013;13:196.

18. Malouf R, Henderson J, Redshaw M. Access and quality of maternity care for disabled women during pregnancy, birth and the postnatal period in England: data from a national survey. BMJ Open 2017;7:e016757.

19. Redshaw M, Hennegan J, Miller Y. Young women's recent experience of labour and birth care in Queensland. Midwifery 2014;30:810-6.

20. Waldenström U, Rudman A, Hildingsson I. Intrapartum and postpartum care in Sweden: women's opinions and risk factors for not being satisfied. Acta Obstet Gynecol Scand 2006;85:551-60.

21. Small R, Roth C, Raval M, et al. Immigrant and non-immigrant women's experiences of maternity care: a systematic and comparative review of studies in five countries. BMC Pregnancy Childbirth 2014;14:152.

22. Institute of Medicine (US). Committee on Quality of Health Care in America. Crossing the quality chasm: a new health system for the 21st century. Washington: National Academy Press, 2001.

23. The Scottish Government. The Healthcare Quality Strategy for NHS Scotland. 2010 http://www.gov.scot/Resource/Doc/311667/0098354. pdf (Accessed 24 Sep 2017).

24. The King's Fund. Improving quality in the English NHS: A strategy for action. The King's Fund website. 2016. Updated 23 February https:// www.kingsfund.org.uk/publications/quality-improvement (Accessed 24 Sep 2017).

25. Reeves R, Seccombe I. Do patient surveys work? The influence of a national survey programme on local quality-improvement initiatives. Qual Saf Health Care 2008;17:437-41.

26. Coulter A, Locock L, Ziebland S, et al. Collecting data on patient experience is not enough: they must be used to improve care. BMJ 2014;348:g2225.

27. The Scottish Government. Scottish Government's Care Experience survey programme. The Scottish Government website. http://www. gov.scot/Topics/Statistics/Browse/Health/careexperience (Accessed 24 Sep 2017).

28. The Scottish Government. 2015 Scottish Maternity Care Survey. Technical report. The Scottish Government website. 2015 http:// www.gov.scot/Resource/0049/00491044.pdf.

29. Scottish Government. Scottish National Maternity Care Survey. The Scottish Government website. http://www.gov.scot/Topics/Statistics/ Browse/Health/maternitysurvey/2015Results (Accessed 24th Sep 2017).

30. Care Quality Commission. Maternity Services Survey 2017. Care Quality Commission website. http://www.cqc.org.uk/publications/ surveys/maternity-services-survey-2015 (Accessed 2 Feb 2018).

31. Redshaw M, Rowe R, Hockley C, et al. Development and pilot testing of the questionnaire for use in the NHS trust based maternity survey. 2007 http://www.nhssurveys.org/Filestore/ documents/MAT07_Development_Report.pdf (Accessed 24 Sep 2017).

32. Scottish Government. The Scottish Index of Multiple Deprivation. The Scottish Government website. http://www.gov.scot/Topics/Statistics/ SIMD (Accessed 24 Sep 2017).

33. Scottish Government. Urban/Rural Classification. The Scottish Government website. http://www.gov.scot/Topics/Statistics/About/ Methodology/UrbanRuralClassification (Accessed September 24, 2017). 
34. Firth D. Bias reduction of maximum likelihood estimates. Biometrika 1993;80:27-38.

35. Gelman A, Hill J, Yajima M. Why We (Usually) Don't Have to Worry About Multiple Comparisons. J Res Educ Eff 2012:189-211.

36. Sawyer A, Ayers S, Abbott J, et al. Measures of satisfaction with care during labour and birth: a comparative review. BMC Pregnancy Childbirth 2013;13:108.

37. Redshaw M. Women as consumers of maternity care: measuring "satisfaction" or "dissatisfaction"? Birth 2008;35:73-6.

38. Coles E, Cheyne H, Rankin J, et al. Getting It Right for Every Child: A National Policy Framework to Promote Children's Well-being in Scotland, United Kingdom. Milbank Q 2016;94:334-65.

39. The Scottish Government. Heat Target (Antenatal Access). The Scottish Government website. http://www.gov.scot/About/
Performance/scotPerforms/partnerstories/NHSScotlandperfo rmance/AntenatalAccess (Accessed $10 \mathrm{Jul}$ 2018).

40. Scottish Government. Inpatient Experience Survey Volume 3: Exploring differences in experience. Scottish Government website. http://www.gov.scot/Publications/2017/04/3075/downloads (Accessed 2 Feb 2018).

41. NHS Scotland, National Statistics. Scottish Patient Experience Survey of GP and Local NHS Services 2011/12. http://www.gov.scot/ Resource/0041/00417119.pdf (Accessed 2 Feb 2018).

42. Crow R, Gage H, Hampson S, et al. The measurement of satisfaction with healthcare: implications for practice from a systematic review of the literature. Health Technol Assess 2002;6:1-244.

43. Sandall J, Soltani H, Gates S, et al. Midwife-led continuity models versus other models of care for childbearing women. Cochrane Database Syst Rev 2016;4:CD004667. 